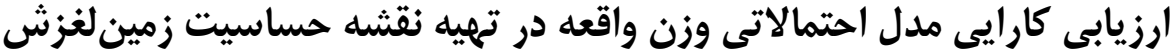

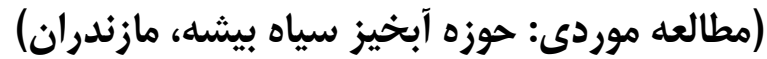

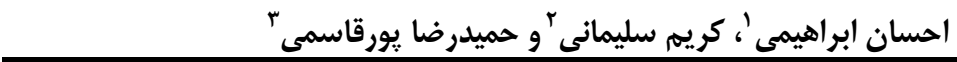

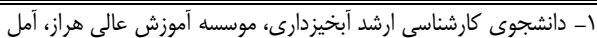

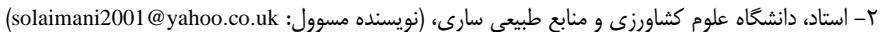

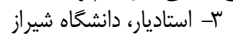 \\ تاريخ دريافت:
}

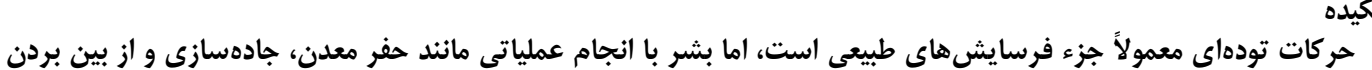

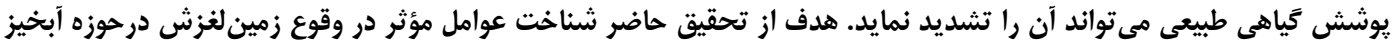

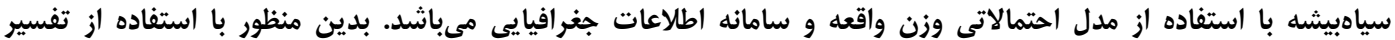

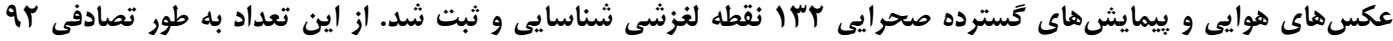

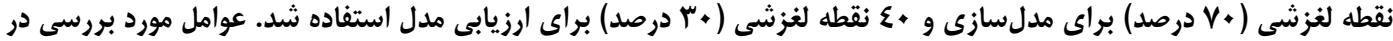

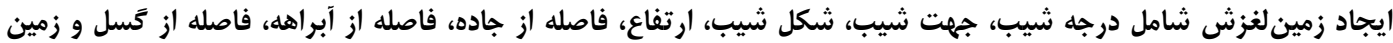

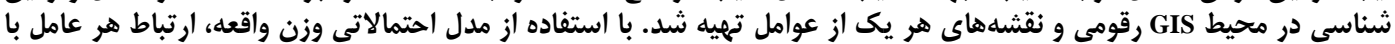

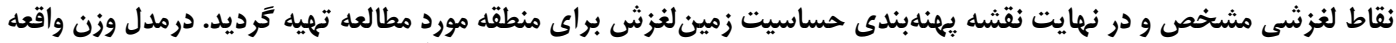

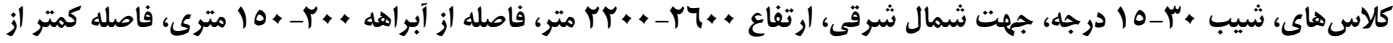

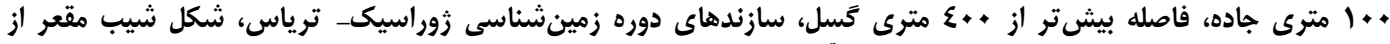

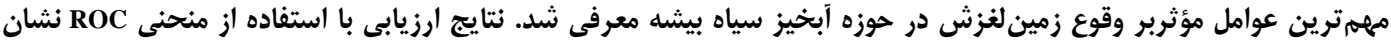

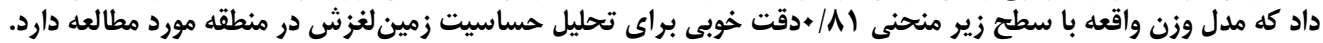

وازههاى كليدى: حركات تودهاى، يهنهبندى حساسيت زمين لغزش، مدل وزن واقعه، حوزه آبخيز سياه بيشه

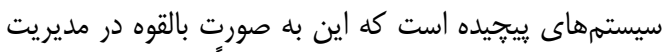

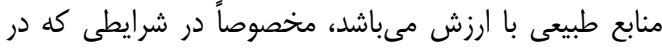

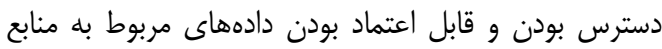

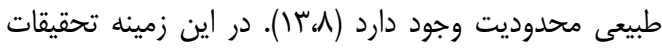

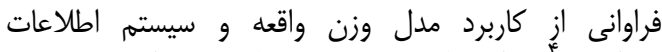

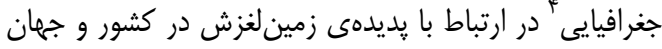

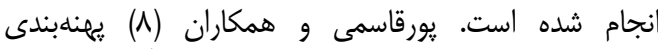

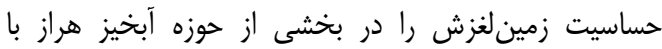

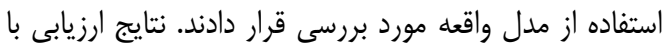

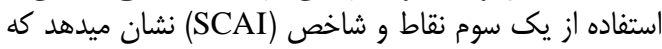

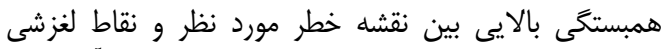

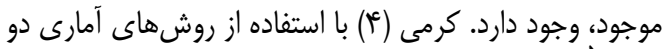

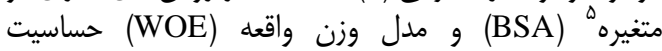

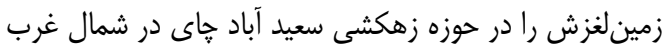

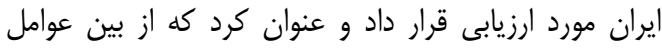

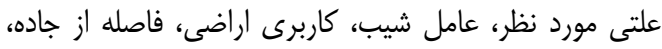

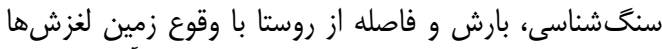

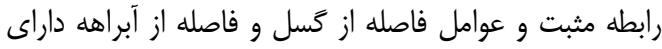

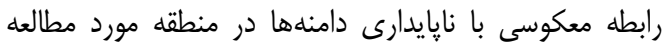

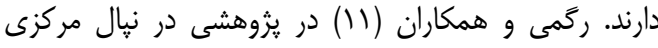

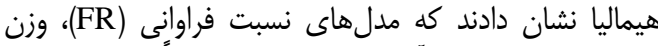
واقعه و شاخصهاى آمارى (SI) نتايج تقريباً يكسانى براى داى داني

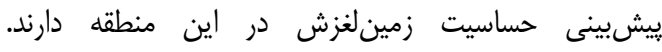
1- Weight of Evidence 4- Geographical Information System

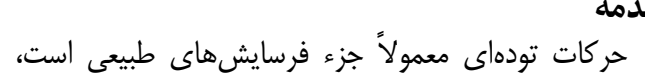

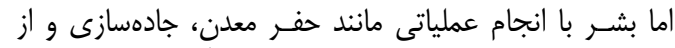

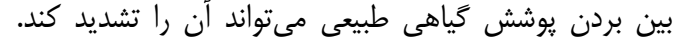

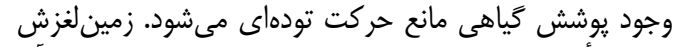

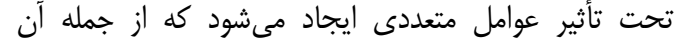

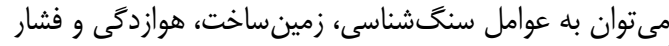

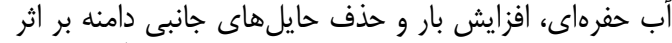

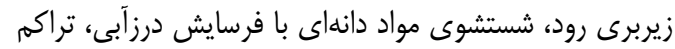

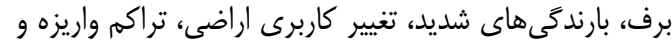

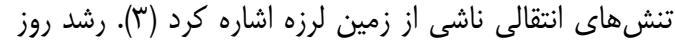

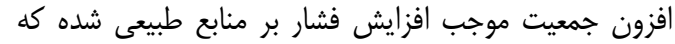

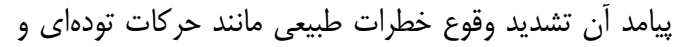

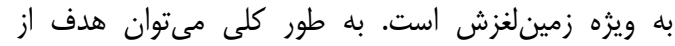

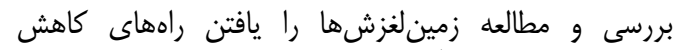

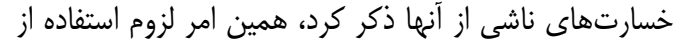

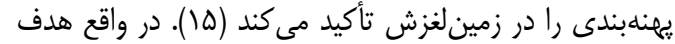

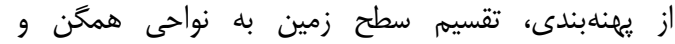

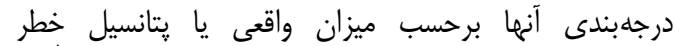

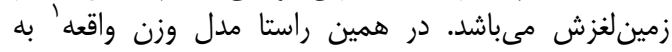

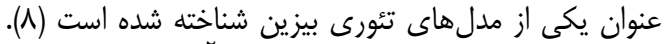

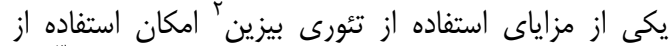

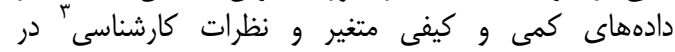
2-Bayesian 3- Expert knowledge

5- Bivariate Statistical Analysis 


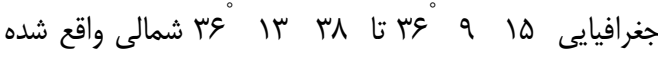

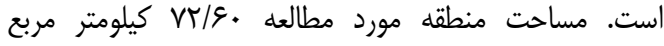

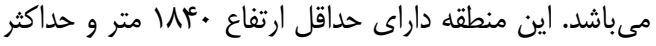

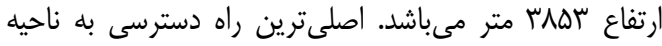

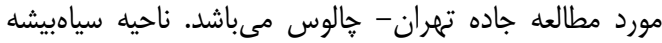

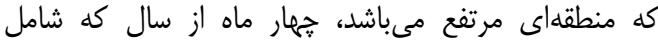

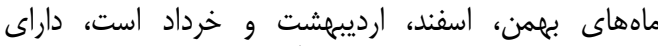

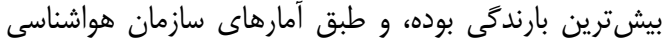

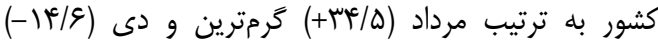

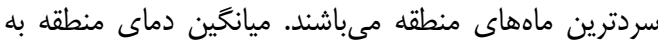

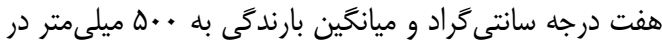
سال مىرسد (شكل ( ).
يورقاسمى و همكاران (•) يكى ارزيابى مقايسهاى بين

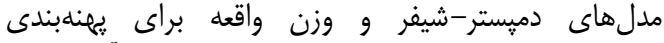
حساسيت زمينلغزش با كمك GIS انجام دام دادند. آنها ارتباط

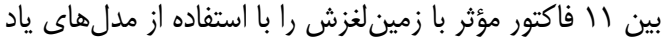

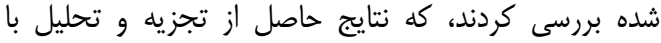

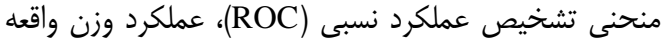

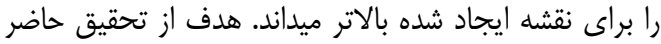

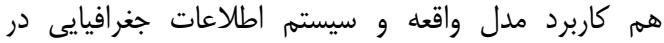

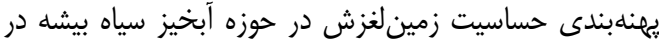

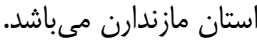

مواد و روشها موردا مورة منطقه مورد مطالعه

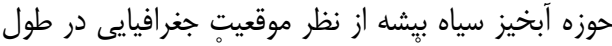

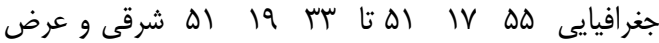

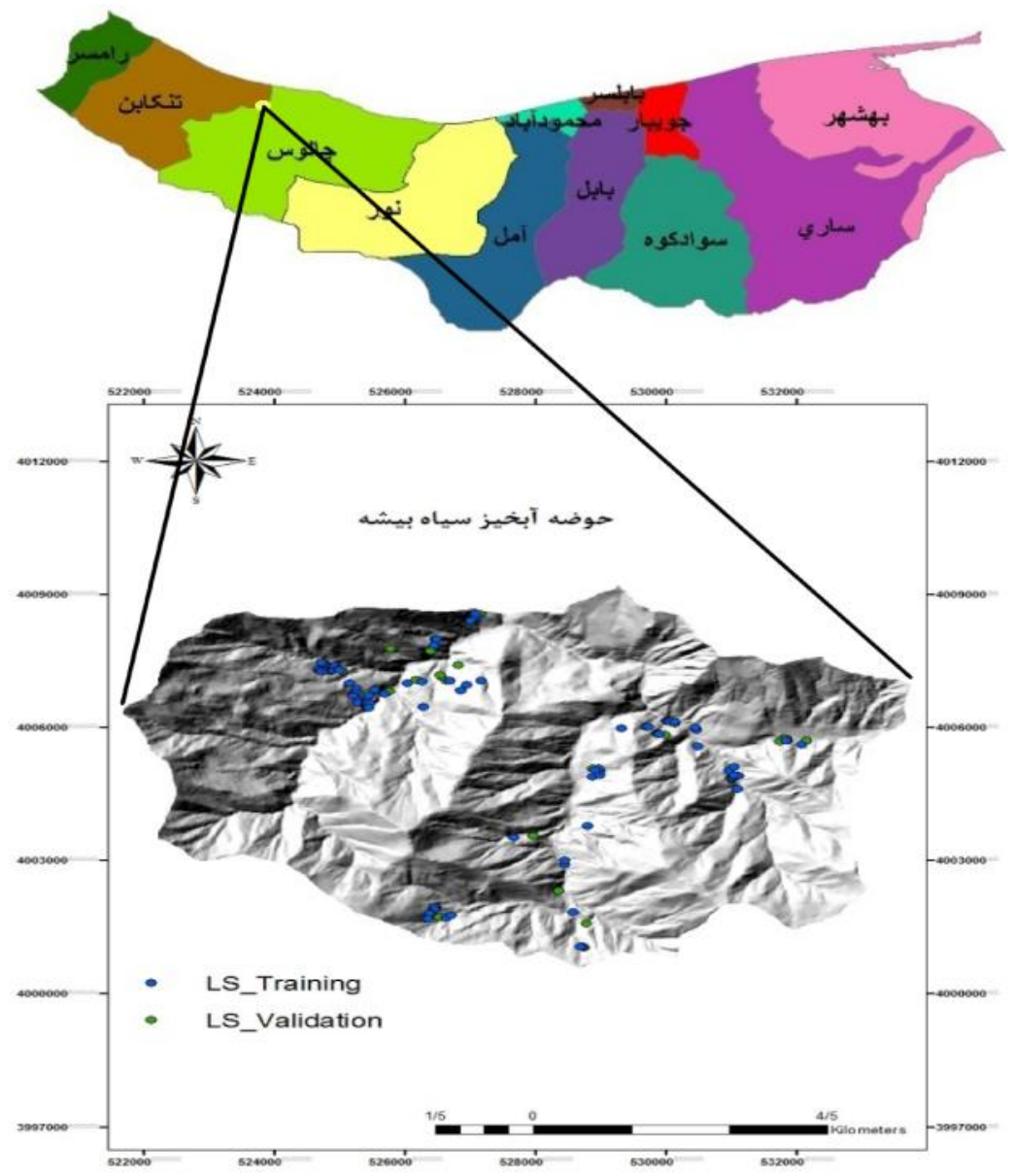

شكل 1- موقعيت منطقه مورد مطالعه

Figure 1. Location of the study area 


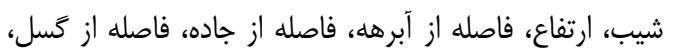

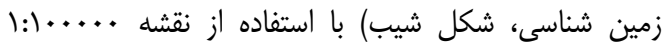

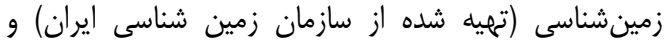

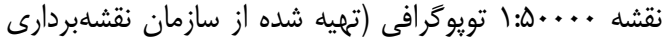

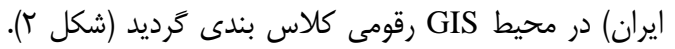

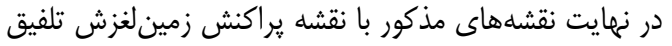

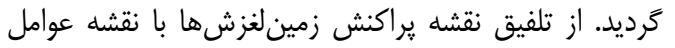

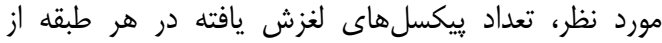
لايههاى مطالعاتى بلهدست آمد.
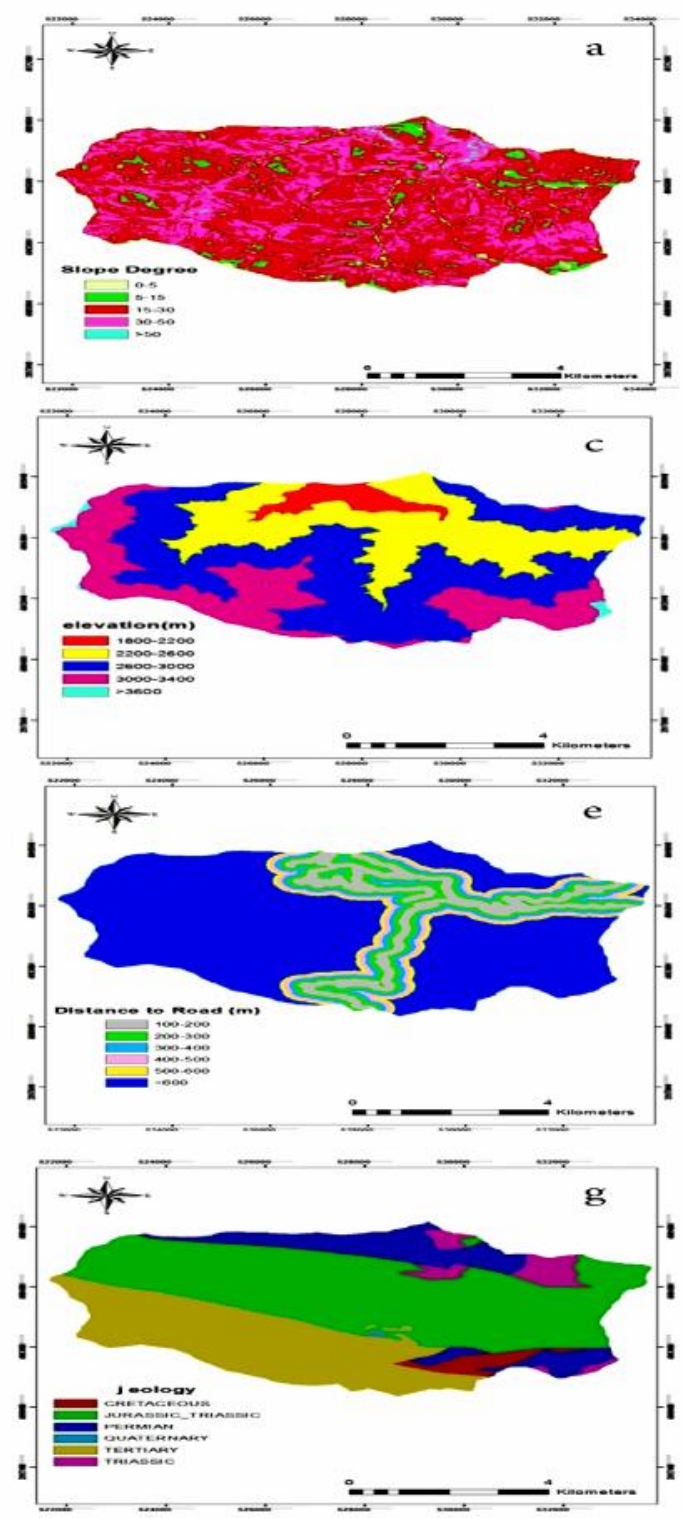

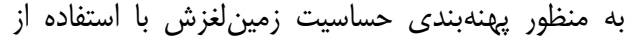

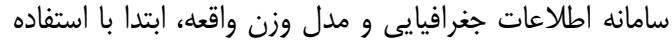

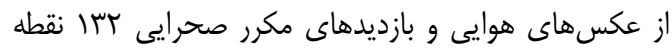

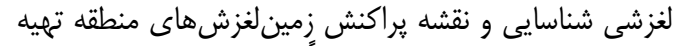

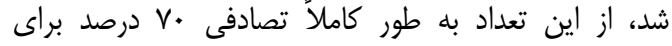

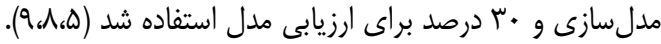

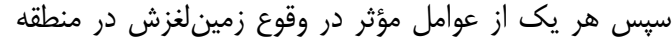
مورد مطالعه شناسايى و نقشهاي مرئ مربوطه (درجه شيب، جهت
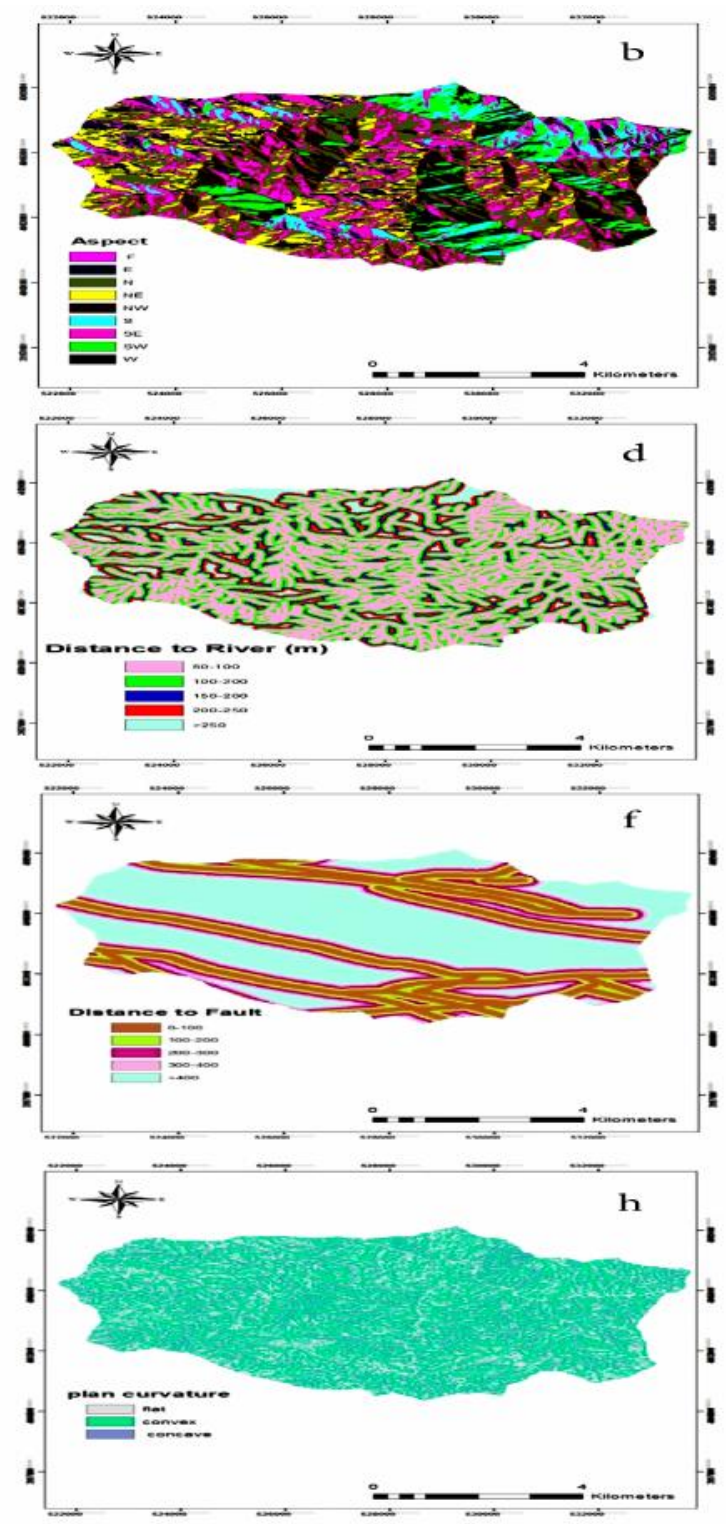

شكل r- نقشههاى عوامل مؤثر، درجه شيب (a))، جهت شيب (b)، ارتفاع (c)، فاصله از آبراهله (d)، فاصله از جاده (e)، فاصله از كسل (f)، زمين

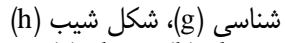

Figure 2. Landslide conditioning factor maps: (a) slope, (b) slope aspect, (c) altitude, (d) distance from river map, (e) distance from road, (f) distance from fault, (g) geology, (h) plan curvature 
N $N_{p i x 4}$

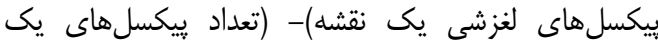
كلاس) + (تعداد ييكسل هاى لغزيكى ديك در يك كلاس).

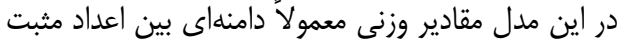

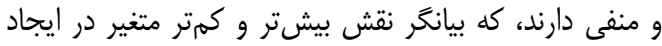
زمين لغزش است. وزن مثبت (Wi

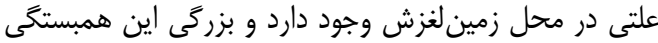

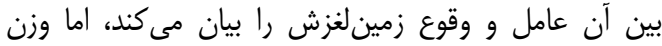
منفى (Wi

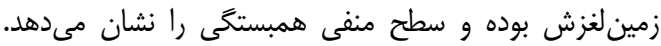

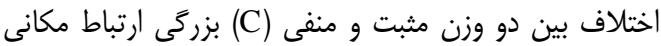

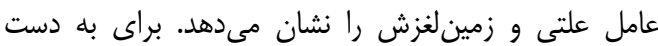

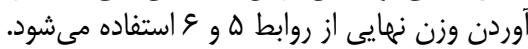

$$
\mathrm{C}=\left[\left(W_{i}^{+}\right)-\left(W_{i}^{-}\right)\right]
$$

$W_{\text {final }}=C / S c$

(†) رابطه (C)

استاندارد : تفاضل وزنهاى مثبت و منفى و

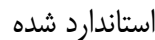

انز : Sc از وزنهاي مثبت و منفى است.

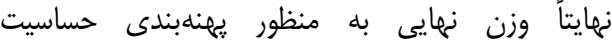

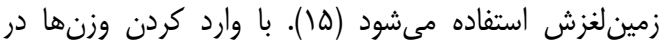

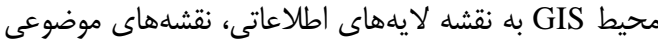

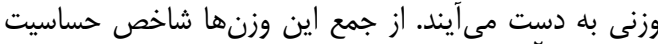
زمين لغزش بَ محاسبه مى دشود.

$$
L S I=\sum\left(W_{f}\right)_{i} \quad(\mathrm{i}=1,2,3, \ldots, \mathrm{n}) \quad \text { (V) }
$$

مدل وزن واقعه

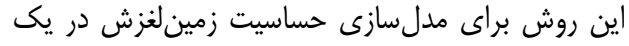

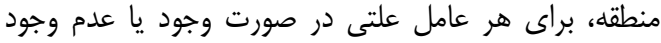

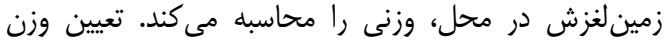

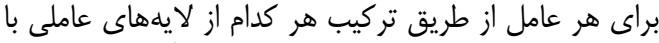

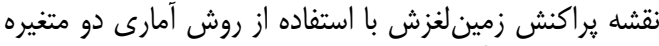

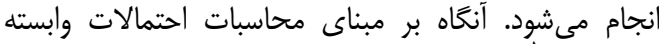

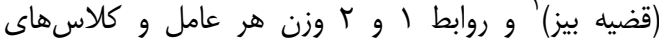
مربوطه به آن تعيين شد.

$$
\begin{aligned}
& W_{i}^{+}=\log _{e}\left[P\left(B_{i} \mid S\right) / P\left(B_{i} \mid \bar{S}\right)\right] \quad \text { (I) }
\end{aligned}
$$

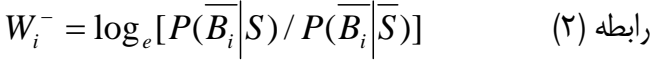$$
\text { كه به منظور درك هرجه بهتر رابطه و سهولت كار به به إنه }
$$

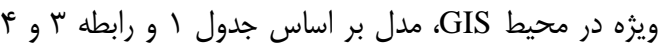

رابطه (r)

$\left.\left.W_{i}^{+}=\log _{e}\left[N_{p i x 1} /\left(N_{p i x 1}+N_{p i x 2}\right)\right) /\left(N_{p i x 3} / N_{p i x 3}+N_{p i x 4}\right)\right)\right]$

رابطه (ا)

$\left.\left.W_{i}^{-}=\log _{e}\left[N_{p i x 2} /\left(N_{p i x 1}+N_{p i x 2}\right)\right) /\left(N_{p i x 4} / N_{p i x 3}+N_{p i x 4}\right)\right)\right]$ N N $N_{p i x 1}$ N N $N_{p i x 2}$ ييكسل هاى لغزشى در يك كلاس)،

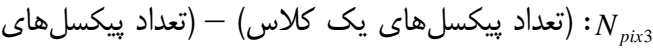

لغزشى در يك كلاس)، (تعداد بيكسل

جدول ا- جهار حالت ممكن از يتانسيل ايجاد زمين لغزش و نقشه زمين لغزش (تعداد يِكسلها = Npix (If)

\begin{tabular}{|c|c|c|}
\hline \multicolumn{3}{|c|}{ عوامل ايجاد زمين لغزش } \\
\hline عدم حضور & حضور & زمين لغزش \\
\hline$N_{p i x 2}$ & $N_{p i x 1}$ & حضور \\
\hline$N_{p i x 4}$ & $N_{p i x 3}$ & عدم حضور \\
\hline
\end{tabular}

Table 1. Four possible combinations of a potential landslide conditioning factor and a landslide inventory map Npix = number of pixels (14)

و و عدم وقوع رخداد (عدم وقوع زمينلغزش) آن است، بيانخر دقت مدل مىباشد.

\section{نتايج و بحث}

نتايج حاصل از ارتباط بين هر يك إن از از عوامل مؤثر با نقاط

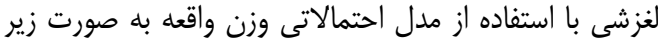
مىباشد و در جدول r ارائه شده است: عامل درجه شيب: نتايج محاسبه وزن (

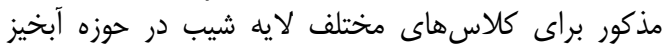
$\sum=W_{f}+1 /$ DV

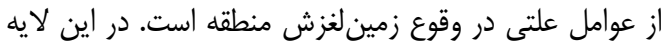

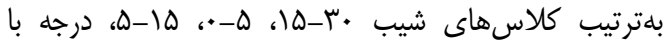

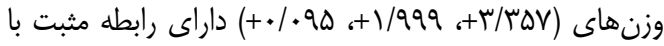

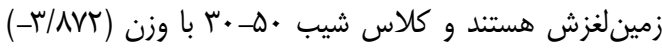

ارزيابى روش يجهنهبندى

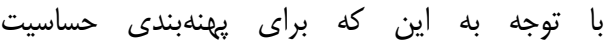

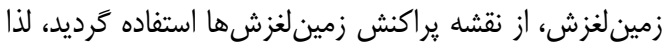

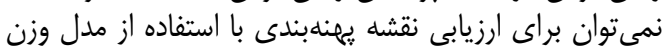

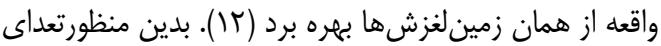

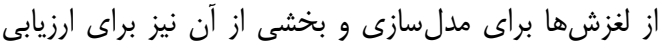

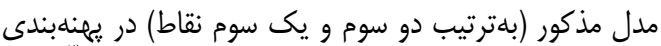

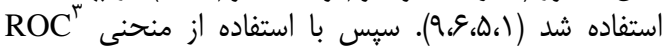

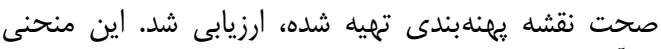

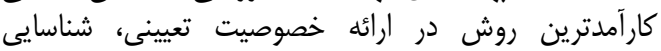

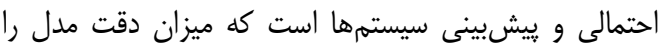

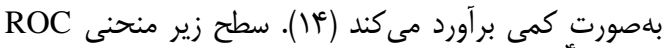
(AUC)

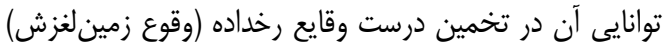
2- Landslide Susceptibility Index (LSI) 4- Area Under Curve 
بدليل تنش برشى يايينتر كه مرتبط با درجه شيب است پايين

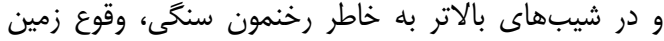
لغزش كم است.
داراى تأثير منفى با زمينلغزش مىباشد. تجزيه و تحليلها

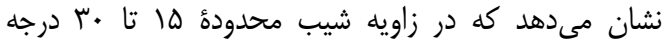

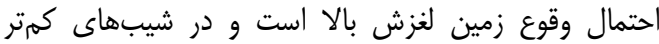

جدول r- ارتباط بين هر يك ازعوامل مؤثر و نقاط لغزشى با استفاده از مدل وزن واقعه Table 2. Spatial relationship between each landslide conditioning factor and landslide by WOE model

\begin{tabular}{|c|c|c|c|c|c|c|c|c|}
\hline$W_{\text {final }}$ & $\boldsymbol{W}^{-}$ & $\boldsymbol{W}^{+}$ & يَيكسل هاى لغزشى & يَيكسل هاى تغزشى & 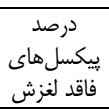 & فييكسل هاقد لغزش & كلاس & عوامل \\
\hline$+1 / 999$ &.$- / . T \Lambda$ & $+\cdot / 994$ & $f / r f \wedge$ & f & $1 / 9$ & Tarr & $\cdot-\Delta$ & \multirow{5}{*}{ شيب } \\
\hline$+\cdot 1 \cdot 90$ & r.|. r & TH & N/\&q६ & $\wedge$ & N/Ftt & IDTA & $\Delta-10$ & \\
\hline$+r / r \Delta V$ & س س\&/. & $+\cdot / 491$ & $s 9 / \Delta \& 9 \Delta$ & $g t$ & D)/\&DT & qभV & $\mid Q_{-}$. & \\
\hline -r/AVr & $+\cdot /$ TA & $-\cdot / v \vee q$ & $\mid V /$ qq & 19 & $r V / q \cdot V$ & gNVqr & $\Gamma \cdot-\Delta$. & \\
\hline • & $+\cdot 1 \cdot \cdot f$ & • & • & $\cdot$ & $\cdot / 4+1$ & $V \times 1$ & $>0$. & \\
\hline$+\cdot|\cdot r|$ & $-.1 \cdot+1$ & $+\cdot 1 \cdot \cdot 9$ & سع./Wו & it & $|r / 9 V|$ & THDF. & مسطح & \multirow{9}{*}{ شيب } \\
\hline$+\cdot /$ \८ & $-\cdot 1 \cdot+i \lambda$ & $+\cdot /$ Irf & TN/TEI & re & $r F / V I I$ & FFAfF & شمال & \\
\hline+ r/rar & צسו/.- & $+\cdot / \& \wedge T$ & Tr/ATE & M & $11 / \Delta \Delta 1$ & r. r. & شمال شرقى & \\
\hline +r/gTI & -.1 .94 & $+\cdot \mid \&$ r. & $\mid V /$ พq| & 19 & $9 / T \& D$ & IENIT & شرق & \\
\hline$-r / .9 T$ & $+\cdot / \cdot V \pi$ & שצא/ו- & $r / I V f$ & r & Q/.r) & letr. & جنوب شرقى & \\
\hline • & $+\cdot / \cdot v q$ & • & • & . & $\mathrm{V} / \Delta \wedge \mathrm{V}$ & ITVA & جنوب & \\
\hline$-1 / \Delta 9 \Delta$ & $+\cdot 1 \cdot 19 q$ & $-\cdot /$ ANV & T/REI & r & V/a19 & אפFE & جنوب غربى & \\
\hline שזו•++ & $-\cdot / \cdot+t$ & $+\cdot / \cdot$ et & N/gQG & $\wedge$ & N/TET & ATIM & غرب & \\
\hline אזץ/I- & $+\cdot 1 \cdot 48$ & $-\cdot / 8 \wedge \mathrm{V}$ & F/TFA & f & אשצ/N & IDSV & شمال غربى & \\
\hline$+1 / \cdot 1$ & TH & $+\cdot / f \cdot f \cdot$ & G/DTY & 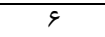 & $r / T \Delta V$ & $V q \cdot V$ & NN*-tr.* & \multirow{5}{*}{$\begin{array}{l}\text { (متر) } \\
\text { ارتفاع }\end{array}$} \\
\hline$+\Lambda / V R r$ & -I/IQT & $+1 / \cdot r \varepsilon$ & $V / / V^{c}$ & $n$ & TV/GNE & שET D. D & r...rE.. & \\
\hline$-\Delta / \Gamma \Delta q$ & 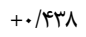 & $-I / T A F$ & $11 / 9 \Delta V$ & 11 & 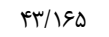 & VArme & rq....... & \\
\hline 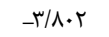 & ונז/•+ & س/VIT & F/MFA & f & $r F / . q F$ & FrVTd & r..._rf.. & \\
\hline • & $+\cdot / \cdot v V$ & • & • & . & .1991 & IrGS & >rf.. & \\
\hline.$- / \cdot 0$. & $+\cdot 1 \cdot \cdot 0$ & $-\cdot 1 \cdot+\Delta$ & Q. & is & $\Delta \cdot / T \& T$ & 9וrा & $\cdot-a \cdot$ & \multirow{5}{*}{ 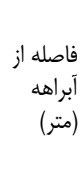 } \\
\hline$+\cdot / 199$ & $-.1 \cdot 11$ & $+\cdot / \cdot r \Lambda$ & $r q / r+\lambda$ & rV & TN/DQ. & 01111 & $0 \cdot-1 \ldots$ & \\
\hline - aftet & $+.1 \cdot 19$ &.$- / 1 r q$ & ৭/v人ץ & 9 & II/TF. & r.rqu & $1 \cdots-10$. & \\
\hline$+\cdot / \varepsilon \Delta \Delta$ &.$- / \cdot 1 \mathrm{~V}$ & $+\cdot /$ TE. & $V / 9.9$ & $\checkmark$ & $\Delta / Q \wedge \Delta$ & 1.181 & ID *-r. & \\
\hline 政 & $+\cdot / \cdot \cdot V$ &.$- / 190$ & T/TQI & r & س س & viar & $>r .$. & \\
\hline$+r / I I \Lambda$ & $-* /|Y|$ & $+\cdot / \& \wedge T$ & $r \cdot / \varepsilon \Delta T$ & 19 & $1 . /$ fet & IN१QT & $\cdot-1 \cdots$ & \multirow{6}{*}{ جاصله از } \\
\hline + & $-.1 .9 \mathrm{~V}$ & שוg/•+ & سع./Wו & it & द/99V & IrG9V & l...r. & \\
\hline$+1 / \Lambda \Delta T$ & $-\cdot 1 \cdot+i \lambda$ & $+\cdot / 9 \cdot r$ & q/VAr & 9 & $\Delta / r \Delta \Lambda$ & qVTF & $r \cdot .-r .$. & \\
\hline 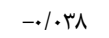 & $+\cdot / \cdot+1$ & -.1 .19 & F/MFA & f & f/erg & $\Lambda \cdot \mu$ & $\mu \ldots-r .$. & \\
\hline- - /199 & $+\cdot 1 \cdot 19$ &.$- / 94$. & $r / I V e$ & $r$ & $F / \cdot f \mid$ & שrm & $f \cdots-\Delta \cdots$ & \\
\hline _r/VVA & $+\cdot / \& V \cdot$ & 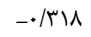 & Q. & is & SN/VRT & $|r E V|$ & $>\Delta \cdots$ & \\
\hline$-1 / 911$ & $+\cdot / .94$ & $-\cdot / \Delta F V$ & $1 \cdot / \wedge V \cdot$ & 1. & IN/VAT & $M F \cdot \Delta F$ & $\cdot-1 \cdot \cdot$ & \multirow{5}{*}{ فاصله از } \\
\hline - T/MM & +.1 .99 & -I/ITK & F/TrA & f & IT/KF & TEtT & n..r. & \\
\hline E & $+\cdot / \cdot r V$ & | & $V / 8 \cdot 9$ & V & $1 . / 9 V T$ & 19915 & 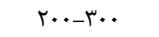 & \\
\hline$+1 / \Delta \Delta \Delta$ &.$- / \cdot \Delta f$ & $+\cdot / 411$ & IF/IT. & سו & $9 /$ & 19998 & $r \cdots-r \cdots$ & \\
\hline$+r / 941$ & $-\cdot / \Gamma \Delta \mid$ & $+\cdot /$ TAT & س F & $\Delta \Lambda$ & FV/OHT & NETE. & $>+\ldots$ & \\
\hline$\cdot$ & $+\cdot / \cdot r \Delta$ & $\cdot$ & $\cdot$ & $\cdot$ & $r / 481$ & FFEV & كر تاسه & \multirow{6}{*}{ زمناسن } \\
\hline س & $-\cdot / V V \Delta$ & $+\cdot /$ \&TD & $\mathrm{V} / \mathrm{IVe}$ & n & $\Delta \cdot / \uparrow a$. & १) $\Delta \Delta F$ & زوراسيك- ترياس & \\
\hline$-t / 1 T$ & $+\cdot 1 \cdot v 9$ & $-\cdot / \lambda \notin \varepsilon$ & $\Delta / \kappa+\Delta$ & $\Delta$ & $I T / \varepsilon \Delta V$ & rrqu. & "يرمين & \\
\hline$\cdot$ & $+\cdot / \cdot \cdot 1$ & $\cdot$ & $\cdot$ & $\cdot$ & . Mft & rat & كواترنرى & \\
\hline$-T / \Delta \& S$ & $+\cdot /$ IAT & $-\cdot / \Delta H_{\Lambda}$ & $|V / r q|$ & 19 & $r q / V v \Delta$ & $\Delta F \cdot M F$ & ترسير & \\
\hline$\cdot$ & $+\cdot 1 \cdot+4$ & $\cdot$ & $\cdot$ & $\cdot$ & F/DIT & 1111 & ترياس & \\
\hline$+\cdot / 994$ &.$- / \cdot r \Delta$ & $+\cdot / T V T$ & $\mid M / \cdot F$ & IT & q/qTळ & $M \cdot r \cdot$ & مقعر & \multirow{3}{*}{ شيب } \\
\hline$+\cdot / \mu \cdot 1$ &.$- / \cdot r \Delta$ & $+\cdot / \cdot t \cdot$ & rq/ır. & ع & $r V / \varphi)$. & GNTAT & مسطح & \\
\hline$-\cdot / \mathrm{MA}$ & +.1 .94 & -.1 .94 & FV/AYS & fr & $\Delta T / F \Delta \Delta$ & 9D।१ & محدب & \\
\hline
\end{tabular}

لغزش بوده و داراى وزن صفر مى باشند، كه مبين اين موضوع

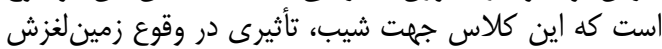
نداشته است.

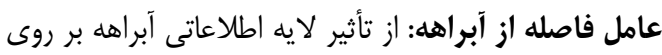

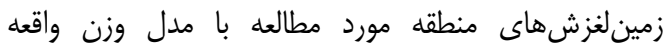

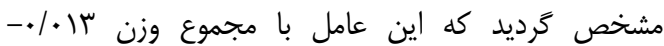

جهت شيب: نتايج ارتباط عامل زمين لغزشها با جهت شيب نشان مى دهد كه جهت شيب با هسم/

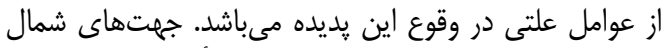

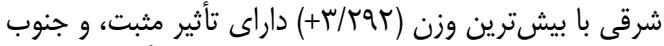

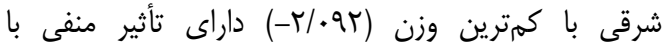

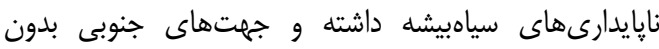


عنوان بارامتر اصلى وقوع زمينلغزش نبوده و عوامل ديخر

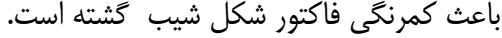

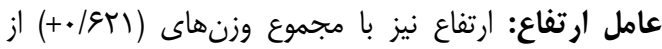

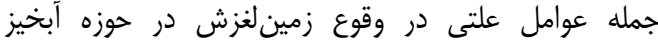

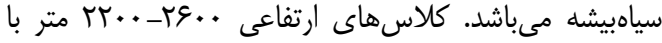

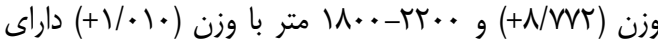

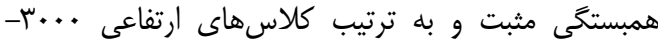

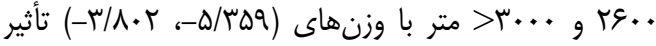

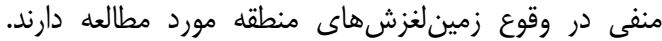

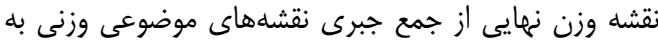

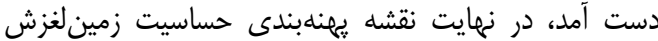

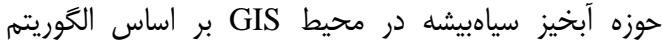

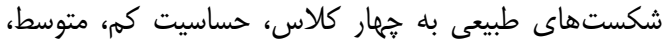

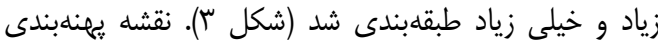

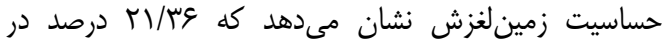

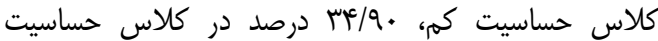

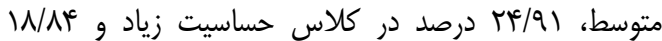

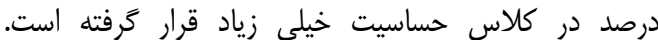

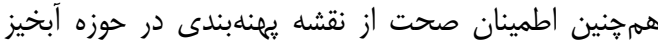

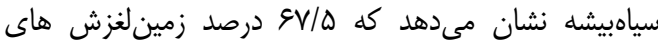

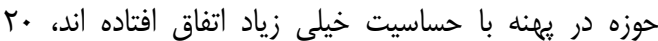

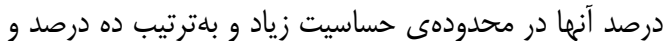

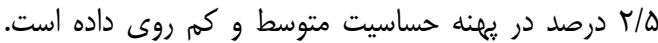

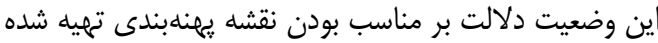

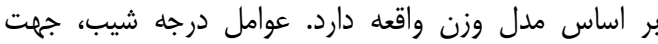

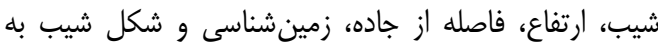

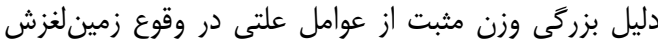

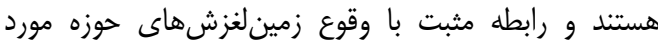

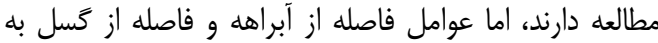

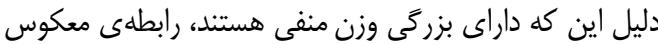

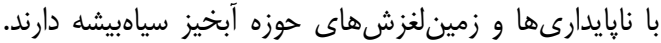

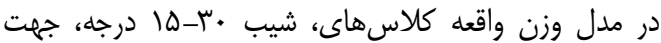

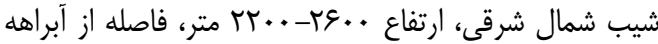

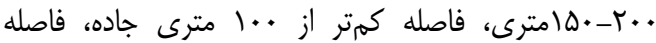

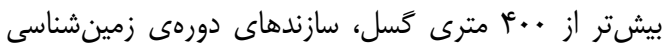

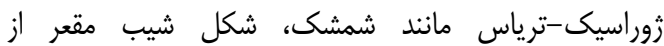

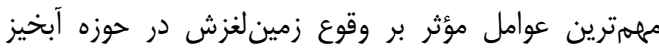

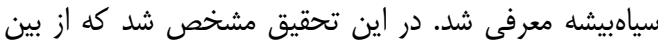

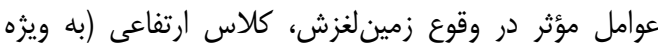

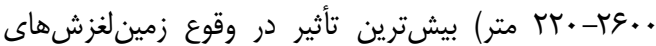

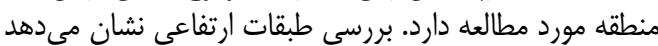

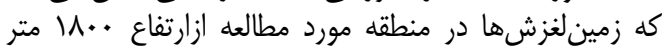

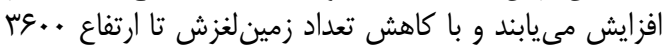

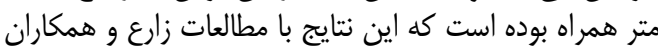

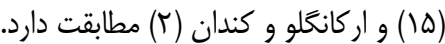

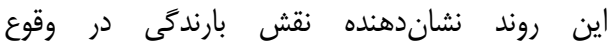

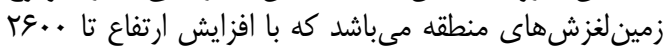

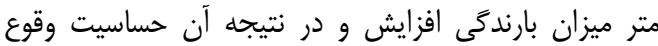
زمين لغزش بالا مىرود. همان طور كه بيان شد بران إن ارزيابى
اين

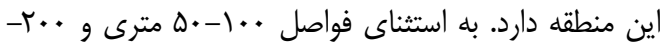

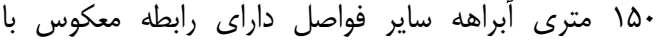
نايايدارىها در منطقه مورد مطالعه دارد.

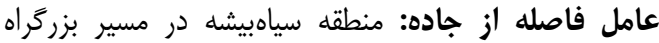

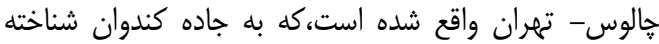

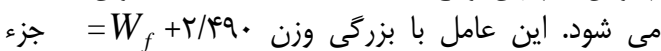

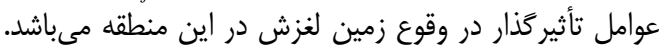

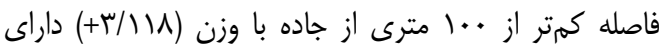

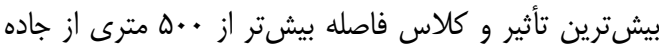

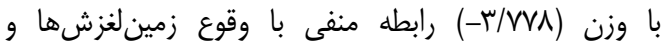

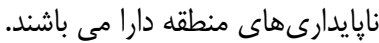

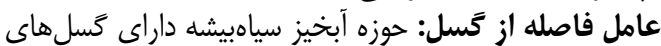

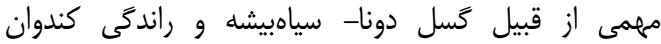

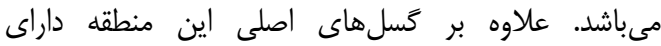

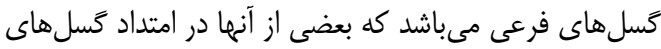

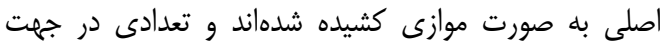

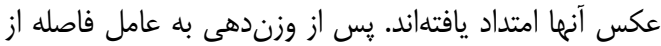

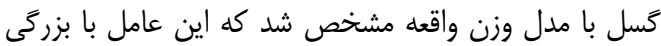

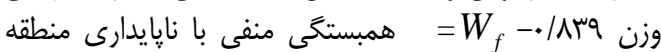

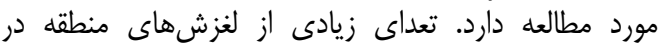

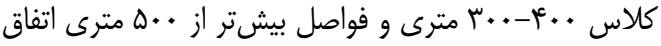

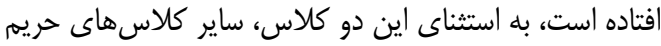

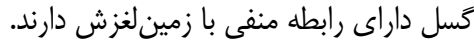

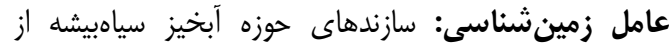

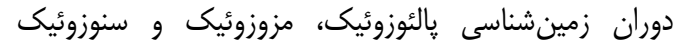

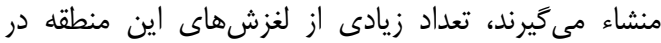

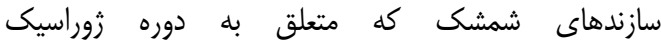

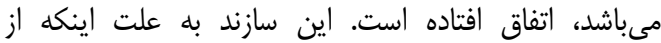

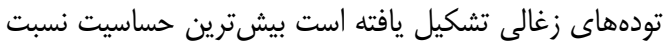

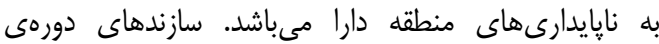

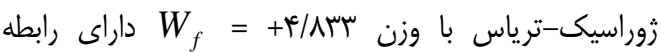

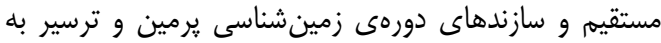

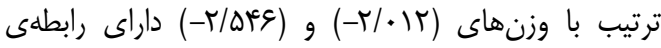

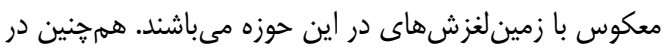

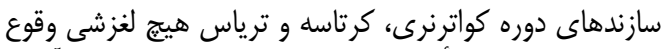

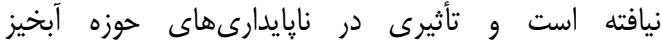

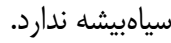
عامل شكل شيب: انواع شكل شيبها به صورد صورت مقعر،

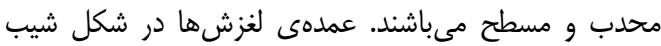

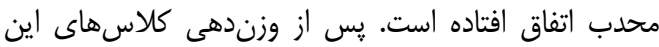

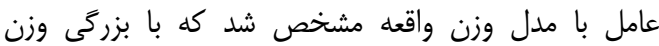

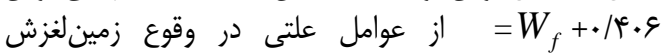

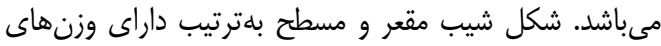

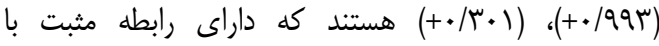

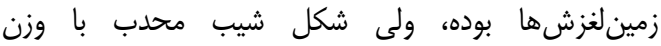
)

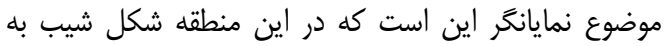


در يزوهش حاضر، نتايج ارزيابى مدل وزن واقعه با استفاده

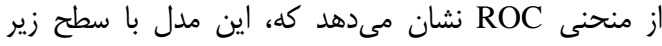

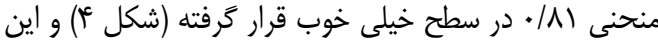

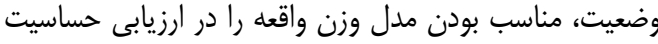

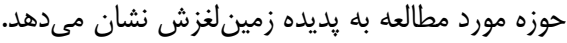

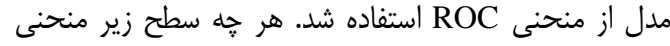

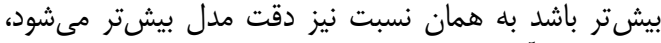

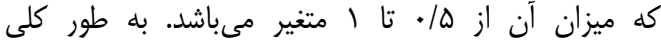

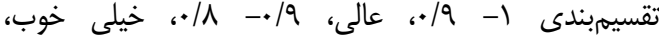

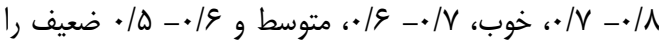

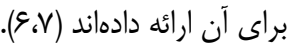

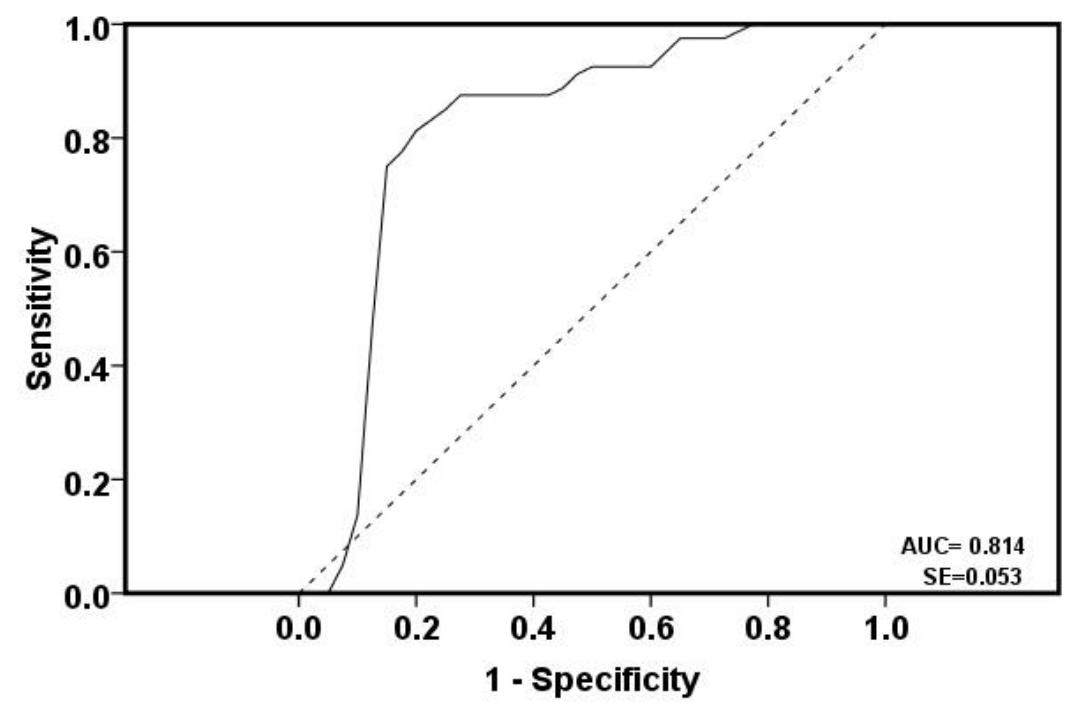

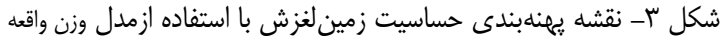

Figure 3. Landslide susceptibility map produced by Weight of Evidence model

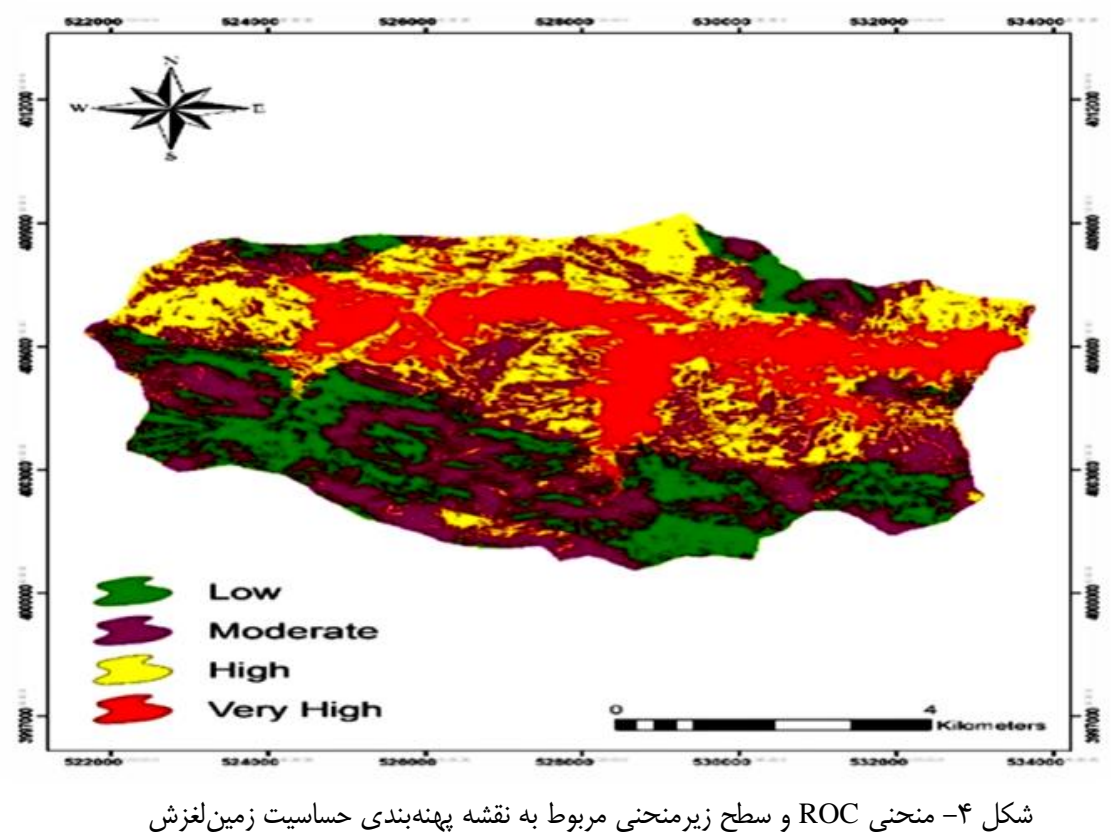

Figure 4. Area under the curve (AUC) and prediction rate graphs for the landslide susceptibility map 
1. Constantin, M., M. Bednarik, M.C. Jurchescu and M. Vlaicu. 2011. Landslide Susceptibility Assessment Using the Bivariate Statistical Analysis and the Index of Entropy in the Sibiciu Basin (Romania). Environmental Earth Science, 63: 397-406.

2. Ercanoglu, M. and G. Candan. 2004. Use of Fuzzy Relation to Produce Landslide Susceptibility Map of a Landslide Prone Area (West Black Sea Region, Turkey). Engineering Geology, 75: 229-250.

3. Feiznia, S., A. Klarestaghi, H. Ahmadi and M. Safaei. 2003. Investigation Effective Parameters in Landslide Occurs and Landslide Hazard Zoning (Case Study: Tejen Dam Watershed). Iranian Journal of Natural Resources, 57: 3-20 (In Persian).

4. Karami, F. 2012. Landslide Susceptibility Assessment Insemiarid Mountainous Watershed, Using Weights of Evidence and Statistical Models (Case Study: Chai Saidabadchai North of Sahand Mountain). Journal of Geographical Research, 106: 44-21 (In Persian).

5. Komac, M. 2006. Landslide Susceptibility Model Using the Analytical Hierarchy Process Method and Multivariate Statistics in Perialpine Sloveni. Geomorphology, 74: 17-28.

6. Moradi, H.R., M. Mohammadi, R. Mostafazadeh and H.R. Pourghasemi. 2010. Landslide Hazard Analysis in Golestan Province Using Dempster-Shafertheory. Quarterly Researches in Earth Sciences, 1: 1-14 (In Persian).

7. Nefeslioglu, H.A., T.Y. Duman and S. Durmaz. 2008. Landslide Susceptibility Mapping for a Part of Tectonic Kelkit Valley (Easten Black Sea Region of Turkey). Geomorphology, 94: 401-418.

8. Pourghasemi, H.R., H.R. Moradi and M. Mohammadi. 2010. Application of GIS and Weight of Evidence Probabilistic Model in the Landslide Susceptibility Mapping. National Conference Spatial Information System, National Cartographic Center, Tehran, Iran, (In Persian).

9. Pourghasemi, H.R., H.R. Moradi, M. Mohammadi, R. Mostafazadeh and A. Aligoli. 2013. Landslide Hazard Zoning Using Bayesian Theory. Journal of Science and Technology of Agriculture and Natural Resources, Water and Soil Science, 16: 109-120 (In Persian).

10. Pourghasemi, H.R., B. Pradhan, C. Gokceoglu and K. Deylami Moezzi. 2013. A Comparative Assessment of Prediction Capabilities of Dempster-Shafer and Weights-of-Evidence Models in Landslide Susceptibility Mapping Using GIS. Geomatic Natural Hazards and Risk, 4: 93-118.

11. Regmi, A.D., K.CH. Davokota, B. Pradhan, H.R. Purghasemi, K. Kumamoto and A. Akgun. 2013. Application of Frequency Ratio, Statistical Index, and Weights-of-Evidence ModEls and Their Comparison in Landslide Susceptibility Mapping in Central Nepal Himalaya. Saudi Society for Geosciences, 7: 725-742.

12. Remendo, J., A. Gonzales, A. Teran, A. Cendrero and C. Fabbri Chung. 2003. Validation of Landslide Susceptibility Maps, Examples and Applications from a Case Study in NORTHERN SPAIN. Natural Hazard, 30: 437-449.

13. Shahabi, M. and A. sadoddin. 2009. The Bayesian Network Approach to Predict the Effects of Drought Management Measures on Dryland Wheat Planted in Golestan Province. 5th National Conference of Watershed Management Science and Engineering, Gorgan, Iran, 3140-3149 (In Persian).

14. VanWesten, C.J., N. Rengers and R. Soerters. 2003. Use of Geomorphological Information in Indirect Landslide Susceptibility Assessment. Natural Hazards, 30: 399-419.

15. Zareh, M., H. Ahmad and S. Gholami. 2011. Landslide Hazard Assessment using of Multi Criteria Decision Making and Geography Information System (Case Study, Vaz Watershed). Journal of Natural Ecosystems of Iran, 1: 156-166 (In Persian). 


\title{
Evaluating the Efficiency of Probabilistic Weight of Evidence Model for Landslide Susceptibility Mapping (Case Study: Siyahbisheh Watershed, Mazandaran)
}

\section{Ehsan Ebrahimi ${ }^{1}$, Karim Solaimani ${ }^{2}$ and Hamid reza Pourghasemi ${ }^{3}$}

1- M.Sc. Student, Watershed Management, University of Haraz, Amol

2- Professor, Sari University of Agriculture and Natural Resources

(Corresponding author: solaimani2001@yahoo.co.uk)

3- Assistant Professor, Shiraz University

Received: March 6, $2015 \quad$ Accepted: June 13, 2015

\begin{abstract}
Mass movements are usually natural erosion, but the human can aggravate it by operations such as mining, road construction and destroying the natural vegetation. The purpose of this study is to identify the factors influencing the occurrence of landslides by using a probabilistic model Weight of Evidence and Geography Information System in the Siyahbisheh Watershed. 132 landslide points are identified and recorded through interpretation of aerial photos and wide field surveys. Randomly out of this number, 92 landslide points (70\%) for modeling and 40 landslide points (30\%) are implemented for evaluation. The factors studied in causing landslide includes slope, aspect, plan Curvature, elevation, distance from the road, distance from the streams, distance from the faults and geological survey using GIS digit and maps are provided for each of the factors. The relationship between each factor with landslide points is determined by using a probabilistic model weight of evidence and the landslide susceptibility map is provided.WOE model introduces classes, 30-15slope degree, aspect of northeast, the elevation of $2200-2600 \mathrm{~m}, 150-200 \mathrm{~m}$ distance from the road, less than 100 meters from the road, distance of more than 400 meters from the fault, the geological formations Jurassic-Triassic, concave curvature as the most important factor causing landslide in the Siyahbisheh Watershed. The results of ROC curve analysis showed that the WOE model with the AUC 0.81 has an acceptable accuracy for landslide susceptibility analysis in the study area.
\end{abstract}

Keywords: Landslide susceptibility Zonation, Mass movements, Siyahbisheh Watershed, Weights-of-Evidence 